\title{
Evaluation brief: Impact on service users of community day services' cessation
}

\author{
Garry Lim
}

\begin{abstract}
INTRODUCTION: This is a brief of a year-long evaluative study and service analysis undertaken by Disability Support Link (DSL), Waikato DHB.

APPROACH: This briefing provides an insight into the evaluative study on the associated impact of cessation of community day services which are key components within the disability sector's service provision. These day services provide an integral outlet of meaningful activities for disabled adults whose current needs impact on their employment. The focus was on analysing repository information over the last decade. The data sources include documented narratives from the needs assessment process and longitudinal resourcing information. In addition, contemporary literature on the role of day activity centres were sourced to identify similar work within this area. The subsequent findings were submitted to the Ministry of Health of Aotearoa New Zealand.
\end{abstract}

FINDINGS: An association was found between service users' lack of access to day services and initial short-term savings to publicly funded disability services budget. However, initial savings were essentially overshadowed by subsequent, increasing, costs over time. There are also associated impacts on disabled adults, such as reports of increasing isolation, decreasing sense of meaningful structured day activities, declining daily functions and possibly resultant maladaptive behaviours. Notably, these issues are not just about funding and resourcing as they impact on disabled people's rights and social connections.

CONCLUSION: This study indicates that cessation and or reduction of day community services derived initial short-term cost savings but subsequent long-term increased support needs requires more costly support packages. Future efficacy lies in further reviewing the positive impact of health-related community day services for people with disabilities; an iteration of day service contracts that produces meaning to the individual and cost efficiency to public health spending.

KEYWORDS: Community day services; Ministry of Health Disability Support Services

AOTEAROA

NEW ZEALAND SOCIAL WORK 31(4), 90-98.

CORRESPONDENCE TO: Garry Lim dsloffice@waikatodhb.health.nz of Health, Disability Support Services.
This is an evaluation brief that was undertaken to develop an understanding of community day activities services' impact on disabled adults, namely service users of community day activities via public funded services from the Ministry
Community day services support disabled adults who cannot find work and improves their personal skills by providing access to regular, meaningful, social contact, and structured and stimulating activities (Ministry of HealthManatū Hauora, 2014). 
In general, publicly funded day services for disabled adults are common in most western countries. Established day services often deliver content on commonly accepted rationales of providing support for carer respite and for disabled adults to have activities, be meaningfully occupied, and with potentially productive social interactions (Health Service Executive, 2018; Whittaker \& McIntosh, 2000).

In Aotearoa New Zealand, communitybased day service centres catering to disabled adults form a well-established, though limited, research area for impact analysis. Anecdotal responses regarding day care service centres often attribute service outcomes to positive conditions in regard to the following: development of functional abilities; increase in daily task independence; ensuring social participation within the community; acceptance of the disabled person's individual rights and offering support to the families (Drăghici, 2015).

On the other hand, tracking and meeting these service outcomes poses a perennial challenge. For instance, a brief overview of service providers' information yields an abundance of data on common daily social activities, such as, ten pin bowling, arts and crafts, computer training and or related therapies, such as yoga (Belfast Health and Social Care Trust, 2018; Luton Council, 2018). These contents are impressive and certainly appeal to many people. A cursory literature search generally supports the importance of day services (Beyer, Brown, Akandi, \& Rapley, 2010; Fleming, McGilloway, \& Barry, 2017; Social Care Institute for Excellence, 2007). Improvements are often categorised within aspects of positive behaviours, mental health and wellness, productivity, family support and community participations. However, there is still a gap on examining outcome measures and accountability for meaningful programmes, structure of content curriculum, and delivery methods and benefits. The absence of specific research in this area contributes to a reduced oversight to justify and account for ongoing positive service development. In service allocation and funding reviews, this aspect is important to continue to ensure public funds are used prudently.

\section{Objectives}

Existing day service contracts within Disability Support Services were the key focus of the evaluation that reviewed and documented accounts of impact of service reduction and cessation within Aotearoa New Zealand. Key objectives of the evaluation included:

(1) Highlight any changes to community day service contract provision in Waikato, New Zealand;

(2) Indicate possible Ministry policy changes influencing service outcomes; and

(3) Identify emergent patterns and demographic trends where possible.

\section{Methods}

Over a span of one year, a mixed methods design was used to evaluate the impact on this clientele. Quantitative data were derived from the SOCRATES system to provide a longitudinal landscape of service resourcing. SOCRATES, a lynchpin to this study, is the database system for the Ministry of Health's Disability Support Services, serving as a national repository of information about service users and service providers (Whānau Tahi Ltd, 2018). The information within the SOCRATES system is entered by Needs Assessment and Service Co-ordination agencies (NASCs) throughout New Zealand and stored for coordination. Numerous SOCRATES longitudinal data of annual resourcing were used to determine the effects of community day service provision on people with disabilities. In addition to SOCRATES, hardcopy disability needs assessment narrative notes provided descriptive information about functions, adaptive needs and usage of community day services in which provision occurred. These 
disability needs assessments or "Initial/ Comprehensive Assessment" documents were sourced from selected service users when they authorised the use of the supply of information for various purposes, such as specialist inputs to help with their assessments outcome, determining health concerns and support services. This includes voluntary consent for use in statistics or research to assist with understanding and improving health services.

\section{Service users}

A key criterion for selected service-users from all of the Waikato region were that they either are currently in or had participated in a Ministry of Health funded day activities services programme in a community setting. Due to resourcing limitations, six cases were selected, with one service user functioning as a control. The service user whose latest resourcing was used as a control is within a banding of the maximum and minimum service resourcing costs of the five other service users. This allows the percentage calculations to better reflect relevance to the general common pool of service users. One highlight to the demography is the clear age difference between the control service user and others; the control case is the oldest (age 70+) in the dataset and has had at least a decade of uninterrupted support from funded services, including day service funding. As such, this person's case represented a useful control against interrupted support from day services.

Service users who have been with DSL for over 10 years were selected to ensure there are sufficient historical data sets to yield data significance and that no new information was created that could influence data integrity. Datasets were then retrieved over their longitudinal service span and interpreted for changes. Numerous records of service coordination, funding allocations and narrative details were tracked as evident in the subsequent subsection; however, only a summary of the key components is released in this brief.

\section{Dataset and analysis used}

The evaluation and service analysis looked into quantitative SOCRATES data and qualitative narrative Initial/Comprehensive Assessment documented information. The control variable for the cohort is the allocation in funded day services for the past 10 years.

The quantitative raw data of disability demographics and funding over the past 10 years were collated from SOCRATES. This included a variety of factual demographics, such as age, gender, ethnicity, and disability diagnoses. More importantly, it also included all changes to funding data during the last decade where applicable. All these datasets were subsequently tracked and recorded in an Excel spreadsheet. The study provided a basic level of analysis of the SOCRATES data to help summarise and find patterns. The focus was on analysing trends in the percentage changes (increases and or decreases) of funding for every single change since the reduction or cessation of day service funding. Each service user would have their full services span mapped on an Excel spreadsheet; this included other $\mathrm{MoH}$ funded services that the person has. The challenge lies in the significant amount of data as funding allocation often have minute changes in lieu of many factors, such as annualising of short-term services, changes to providers, adding new services, or moving to other types of service. To facilitate the process more efficiently, key funding periods were identified:

1. Start of day services dates

2. Start of partial reduction of day services

3. Start of full cessation of day services

4. Resulting funding savings in the short term

5. Long-term funding changes to all other services. 
A capture of the trends of percentage differences over time was utilised to calculate the total number of resourcing increases for each of the selected service users. Inferential statistics were not used given the small sample size nor is there a need to go beyond current percentage analysis of absolute values to determine or pursue association.

The qualitative aspect of the analysis commenced with the request and recording of all narrative documents around those identified times of significant funding changes reflected in the initial quantitative review. The content of the narrative data from the needs assessments and reviews provided contexts on a variety of disability aspects, such as recent living situations, communications, behaviour, cognition changes, physical functions, sensory functions, mobility at home and or out in the community, family whānau contacts, culture and key meaningful goals.

The objective was to analyse this recorded information in the hope of deriving associations or rationales for the reason for changes in funding. The study looked for meaning and determines if some pieces of narrative data had value; a helpful aspect was to focus on data records around the key funding periods changes highlighted earlier. The study then sought to categorise the data; this included word indexing of the needs assessments and review of narrative data. The strategy was to look for patterns and themes around behaviours, caregiving interactions, specialist inputs and other possible ideas. Subsequently, scrutiny was applied to identify possible themes and look for common changes in disability support needs that occurred around the time of funding changes. A constant crossreferencing of service allocation data from SOCRATES and narrative assessment information was applied throughout the whole process to try best to interpret the data and determine their relationships. The process of cross-referencing a decade's worth of SOCRATES data and paper-based needs assessment information was ostensibly taxing but necessary to derive rationale for decade-old historical developments in relation to cessation of day services and its impact.

In addition to SOCRATES data, a service provision allocation tool ICARE was utilised for applicable service users. The ICARE tool is a $\mathrm{MoH}$ and contracted provider mechanism that lends the means to calculate and determine weekly support hours for users who are in funded group home settings. Using this tool, the evaluation compared the changes in support hours over time for the control service user and for service users who had changes to their day services funding. This is done by accessing the ICARE tool which translates the assessed needs of a disabled person into daily and weekly hours of direct support required to support the person in a community residential setting. The use of the ICARE tool is not applicable to all the service users as it relates to service users in group homes and/or in certain, specific, community-based residential-like settings.

\section{Ethical considerations}

All services users and or proxy carers, such as family members or support staff provided informed consent for their information to be used for statistical and/or research purposes. This includes an authorisation of information to be used for statistics or research where users will not be identified.

This briefing omitted most demographic information to maintain confidentiality. The detailed evaluation contained information such as, gender, race, diagnoses, and medical ailments, other social and economic details. There are no restrictions on the publication of this evaluation and there are no conflicts of interest. Clarifications were sought from the Research Office, Quality and Patient Safety, Waikato District Health Board to confirm that the ethics committee need not be involved for the purpose of this evaluation exercise. 


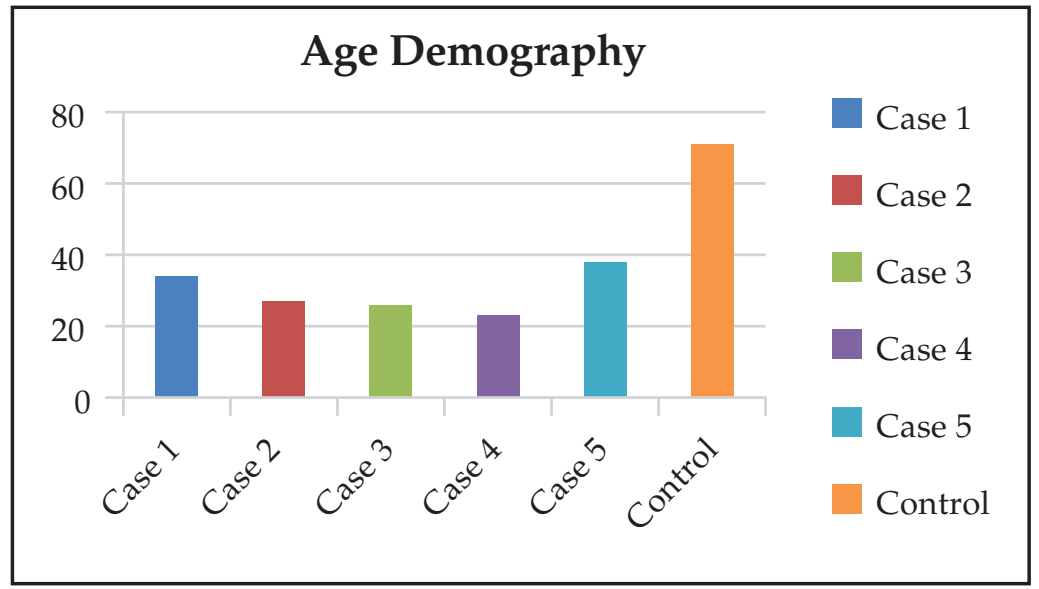

Figure 1. Age

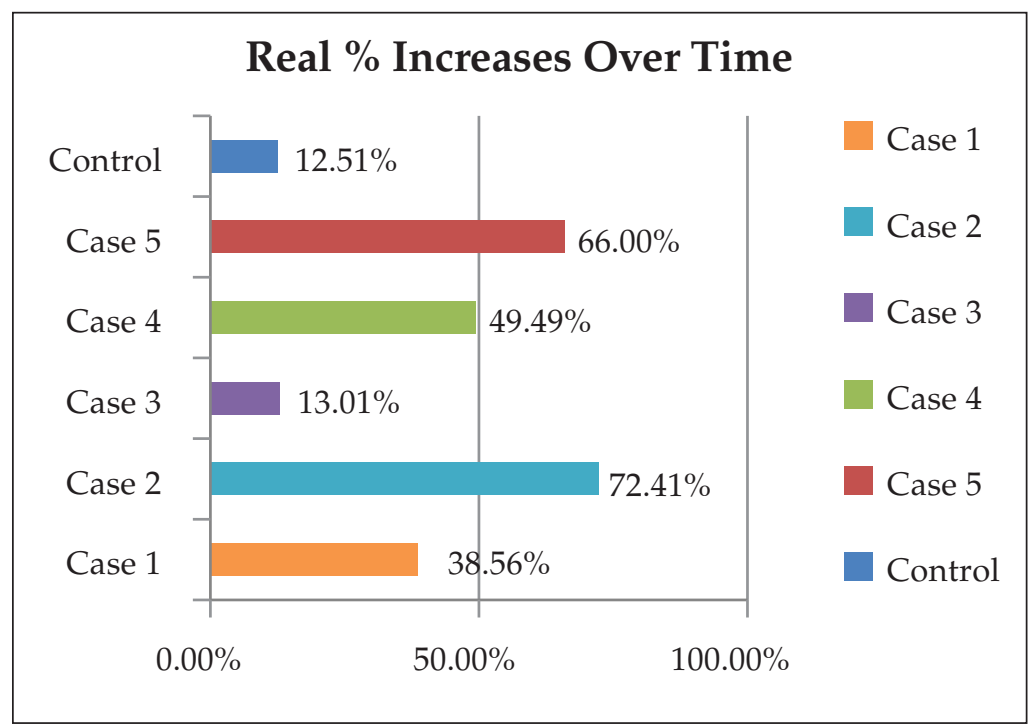

Figure 2. Percentage increases over time.

\section{Findings}

The study found associations between the cessation of funded day services, subsequent increases in support needs and resourcing allocations within the cohort. Various graphic presentations will be used to provide a summary of the findings. Figure 1 provides an overview of the age demographics of service users presented in this evaluation.

The evaluation noted that most service users were below 40 years of age when their services ceased or were reduced. It was mostly due to service users' changes in support needs identified in the narrative information. There were initial cost savings that correlated to these periods of change. Apart from these cases, the service user identified here as the control case has consistent day service funded and continues to receive this service as of the date of the evaluation. All the other cases had services ceased permanently at some point of service provision.

The sample included people in their 20s to those more than 70 years of age. This age range is an optimal depiction of the common pathway for school-leaving disabled adults who require support to continue to have meaningful day activities once they reach school-leaving age. The control case's age provided an observation that participation levels in meaningful day activities do not routinely reduce in advanced age; despite people requiring increased care and support around other health needs. The later sections will use the control case's service allocation as a possible predictive indicator for other services users if there were no changes to their day service allocations.

From the needs assessment narratives, the control service user resourcing has had little impact from common significant issues such as neither major health concerns nor injuries sustained. Further discussion on this aspect will take place in the next section. The next figure provides a graphic presentation of the selected service users' overall resourcing trends and increases for Disability Support Services over time.

All service users exhibited increases in support over time but at different rates, as shown in Figure 2. The percentage resourcing changes were derived from each service user's personal resourcing pathway and mapped the service users' percentage changes beside each other. This evaluation omitted the use of exact resourcing allocations of the individual due to the premise that each starting needs 
and resourcing point for each service user is individualised and unique. Instead, the evaluation focused on using percentage changes as they are useful for comparing the funding increases where the initial starting points and totals are different.

The service users' needs assessment narratives were cross-referenced around those periods of time when cessation occurred. It was found that the control service user continues to be well supported with day support services. Although the control service user had preexisting maladaptive behaviours, these were well managed within the current day services. As a result, the support resourcing increases for the control case were at the lowest among the cohort. For the other five service users who had services ceased or reduced, there were increased recorded narratives of maladaptive behaviours that carers found challenging to safely support; for instance, records showed that some service users required long-lasting behaviour support specialist involvement to manage safe service. The carer's capacity to provide support has changed or is insufficient to meet the needs of the person after cessation and safety issues became apparent. These challenges in behaviours persisted over time and resulted in increasing support needs funding.

The findings show that the control service user, represented in blue, has the least percentage increase in support allocations over time $(12.5 \%)$. Increases in support services costs for the other five service users when day service funding was removed or reduced (Figure 2). Over time, the initial cost savings from day service reductions were overshadowed substantially by the resulting higher resourcing costs. From this evaluation, the increase ranged from 13\% to $72 \%$. Both calculated mean and median cost of the five service users were at nearly $48 \%$ above their original funding. If the evaluation was to assume the control service user's pathway as a comparison, this is well over the common resourcing progression of $12.5 \%$. The bar chart presented a surge of resourcing costs which relates to increasing support needs for service users. There is expressed possibility that removal and reduction to funded day services resourcing could impede on subsequent cost-efficient service provision.

\section{ICARE tool}

In determining associated factors for the increased needs of the service users, the analysis reviewed the daily and weekly hours of support that some of the service users were allocated via the data available in ICARE. As identified in the methods section, not all service users go through this process. The ICARE data are available for four service users, including the control service user. The

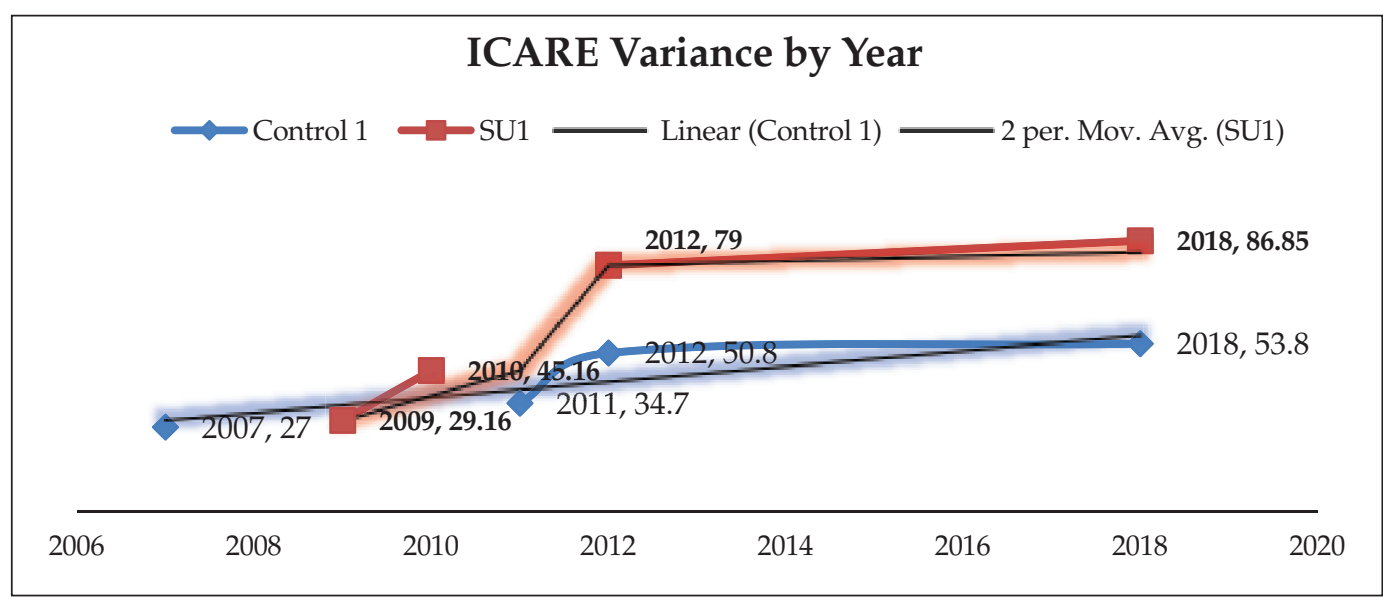

Figure 3. ICARE variance by year. 
other two service users do not require the use of this tool in lieu of their specific service types in the community.

Figure 3 demonstrates a comparative case study of the trajectory of ICARE tool hours datasets allocation for the control service user and one service user. The graphical presentation shows a comparison of ICARE hour's changes as the years of supports continues; it shares a snapshot of a control service user with current day service resourcing allocations, versus a service user who has had day services resourcing withdrawn.

In Figure 3, the control service user represented in blue is plotted over a 10year service span; likewise, the same method is done using service user one in red indices over an eight-year service span. The difference in years is due to service users' date of entering service and has little impact to the analysis given both are within the five- to 10-year service range. There is a distinguishable gradual sloping linear resourcing pathway for the control service user which indicates stable progression. Incongruous to the same timeline, service user one (the red coloured line) had steep surge increases in support hours. Service user one would appear to break away from the common pathway of stable progression determined from the control service user's 10 -year pathway. Narrative inputs indicate that during this 10-year period, the blue control case is settled and well adapted while supported to live as independently as possible. However, this was not the case for service user one. Cessation of day service was associated with an increased in support needs relating to behaviour challenges that impacted on the needed support hours.

The need to have added supports to manage increased disruptive behaviours may indicate that the person is not coping, nor emotionally stable. This aspect will have a consequential impact on the quality of life for the service user and the person's immediate peers and support environment.
Using the above comparison, this study shared associations that funded day service reduction precipitates a short-term decrease in cost savings but an increase in ICARE support hours over the next eight years.

\section{Discussion}

\section{Service improvement}

There were insightful details uncovered in the lived experiences of disabled adults from a lifespan perspective of service allocations within community day activity services that are funded. This analysis benefited from using the system's existing mechanisms to inform publicly funded health services for forecasts and to better meet service users' needs. In completing this service analysis, the Ministry would benefit from the information to determine and outline longitudinal changes to the person and corresponding service allocation. Simultaneously, this exercise surfaced possibilities for positive avenues to better meet the individual's occupational needs and which are cost effective. The findings are conclusive, cessation from funded community day services results in an increase in resources required over time. The presumed initial cost savings overran in tandem to increasingly more challenging support needs, such as more challenging behaviours, complex care routines, need for increased oversight and supervision.

\section{Consequences of the cessation}

While causation cannot be implied, the concern remains that dedicated day service cessation resulted in many issues. This includes increases in isolation, decreases in functional activity of daily living, increasing user's needs and overall increase in long-term costs to the Ministry. These are systemic concerns for everyone involved including family and whānau, professional staff and direct support workers. From the qualitative analysis, there are recorded narratives of increasingly stressful and untenable daily supports from paid and or familial carer supports. 
Some of the key features from the study reveal new learnings on the trends of service packages of service users with an omission to day service (be it complete withdrawal, non-instatement in past records or reducing units). Currently new and current service users with Ministry of Health services are unable to access $\mathrm{MoH}$ funded new day service units. Service users instead access Ministry of Social Development programmes that are limited to certain providers, have limited number of days in attendance and are limited by concerns of available resources within the specific locality.

This study suggests an association between service users losing access to day services and short-term savings to disability services budgets. However, findings from the longitudinal comparison suggest that, over the years, their need for more individual supports essentially overshadowed all other cost savings. The narratives in the findings found that behaviours become increasingly maladaptive and untenable for carer supports. This study provides a documented association, albeit at a scaled version, that links the result of short-term savings to long-term increased support needs requiring more costly (some exponentially more) support packages to budgets.

Cessation of funded day services creates a concerning long-term implication for the individual and subsequent health care system. Longitudinal case comparison data illuminated a clear phenomenon whereby service users were found to present with more severe emotional problems and challenging behaviour when Disability Support Services funded day services were withdrawn, reduced or ceased. This resulted in a slow, but traceable, long-term higher cost to Disability Support Services when maladaptive behaviours increasingly become more challenging to manage in service provision and coordination. This year-long study has yielded well documented associations; however, existing and persistent logistical challenges limit the capacity to infer and present this topic area in greater detail.

\section{Conclusion}

This brief evaluation highlights that day services play a role in enhancing service allocation outcomes and the cost-stabilising effect illustrates a positive investment outcome via day service involvement. The findings from this evaluation demonstrated that cessation of service also had higher rates of negative social and personal effects, such as a persons' increased isolation, a decrease in day activities, a decrease in functions and an increase in maladaptive behaviours. The rate of negative effects associated with loss of day services indicates an overall increase in long-term Ministry spending. This article stresses that an action plan to review further cessations needs to be in place to start mitigating these concerns. This will likely decrease or, at a minimum, stabilise the population's resourcing. In conclusion, there is a clear need to further evaluate current outcomes of existing day services. One possibility could be replicating this evaluation in different regions of Aotearoa New Zealand and track similar practices before cessation of day services occurs.

Acknowledgements: Kindest regards to Barbara Walters and Graham Guy at Disability Support Link for providing sound advice and knowledge to the subject matter. This endeavour was made possible through the helpful contributions from staff at Disability Support Link, Waikato District Health Board.

Note: Ministry of Social Development (MSD) currently provide resourcing of vocational supports and service via MSD-funded providers.

\section{Correspondence}

Please direct any correspondence to Disability Support Link, Waikato District Health Board, 69 Rostrevor St, Hamilton 3204, New Zealand (email: dsloffice@waikatodhb.health.nz). 


\section{References}

Belfast Health \& Social Care Trust. (2018). Day centres - physical and sensory disability.

Retrieved from http://www.belfasttrust.hscni.net/ DayCentresPhysicalSensoryDisability.htm

Beyer, S., Brown, T., Akandi, R., \& Rapley, M. (2010). A comparison of quality of life outcomes for people with intellectual disabilities in supported employment, day services and employment enterprises. Journal of Applied Research in Intellectual Disabilities, 23(3), 290-295. https://doi.org/10.1111/j.1468-3148.2009.00534.x

Drăghici, E. (2015). The importance of day care centers in the life of the disabled people and their families. Social Work Review / Revista de Asistenta Sociala, 14(3), 137-151.

Fleming, P., McGilloway, S., \& Barry, S. (2017). Day service provision for people with intellectual disabilities: A case study mapping 15-year trends in Ireland. Journal of Applied Research in Intellectual Disabilities, 30(2), 383-394.

Health Service Executive. (2018). Time to move on from congregated settings a strategy for community inclusion. Retrieved from https://www.hse.ie/eng/services/list/4/ disability/congregatedsettings/timetomoveon.html

Luton Council. (2018). Adult day services-Luton Council. Retrieved from https://www.luton.gov.uk/Health_and_ social_care/adult_social_care/disabilities/Pages/Adultday-services.aspx

Ministry of Health-Manatū Hauora. (2014). Community day services. Retrieved from https://www.health.govt.nz/ your-health/services-and-support/disability-services/ types-disability-support/community-day-services

Social Care Institute for Excellence. (2007). Communitybased day activities for adults with learning disabilities. Community Care, (1677), 32-34.

Whānau Tahi Ltd. (2018). Whānau Tahi | Socrates—enabling services for those with disabilities. Retrieved from http://www.whanautahi.com/simplhealth/122-socratesenabling-services-for-those-with-disabilities

Whittaker, A., \& Mclntosh, B. (2000). Changing days. British Journal of Learning Disabilities, 28(1), 3-8. https://doi. org/10.1046/j.1468-3156.2000.00024.x 